\title{
Comparación de infecciones por Pneumocystis jiroveci en pacientes con y sin diagnóstico de infección por VIH
}

\author{
Alejandro Rey, Cecilia Losada, Julieta Santillán, Fernando Fiorentini, María Schiaffino, \\ Héctor J. Peroni, Diego Giunta, Laura Barcán y Gabriel Waisman
}

\section{Pneumocystis jiroveci infection in patients with and without HIV: A comparison}

Background: Respiratory infection caused by Pneumocystis jiroveci is a common opportunistic infection in patients with human immunodeficiency virus (HIV) with CD4 counts $<200$ cells $/ \mathrm{mm}^{3}$. However, it has also been reported in patients with other causes of immunosuppression. Objectives: To compare the characteristics, severity and mortality of respiratory infection by $P$. jiroveci in patients with and without HIV infection. Methods: Retrospective cohort follow-up of adult patients admitted to our hospital with infection by P. jiroveci since 2006 to 2013. Results: We included 82 patients with respiratory infection by $P$. jiroveci of which $55 \%(45)$ were not infected with HIV. In this group, $68.8 \%$ (31) had diagnosis of cancer and 20\% (9) received solid-organ transplant. 57.9\% (26) were hospitalized in an intensive care unit. 42.2\% (19) suffered multiple organ failure (MOF), 46.7\% (21) required mechanical ventilation (MV) and 40.9\% (18) inotropic drugs. Mortality was 33.3\% (15). Statistically significant differences were observed between groups in age $(\mathrm{p}<0.001)$, requirement of $\mathrm{MV}(\mathrm{p}<0.001)$ inotropic drugs ( $\mathrm{p} 0.001)$ and MOF $(\mathrm{p}<0.001)$. Mortality was higher in the HIV-positive group, reaching statistical significance ( $\mathrm{p}$ 0.007). Conclusion: Pneumocystis pneumonia mortality was higher in patients without HIV, who suffered more complications and progression to respiratory failure with MOF.

Key words: Pneumocystis jiroveci, opportunistic infections, immunocompromised, HIV, respiratory tract infections.

Palabras clave: Pneumocystis jiroveci, infecciones oportunistas, inmunocomprometido, VIH, infecciones respiratorias.

\section{Introducción}

$P$ neumocystis jiroveci es un hongo que ocasiona infecciones respiratorias en pacientes inmunocomprometidos, clásicamente descrito en individuos portadores del virus de la inmunodeficiencia humana (VIH) con recuento de LTCD4 < 200 céls. $/ \mathrm{mm}^{3}$. Sin embargo, también se han reportado casos en pacientes con otras causas de inmunosupresión, como tratamientos con agentes antineoplásicos, inmunosupresores o glucocorticoides, y en quienes padecen enfermedades onco-hematológicas $^{1-5}$.

La incidencia de neumonía por $P$. jiroveci aumentó paralelamente a la expansión de la pandemia de VIH, constituyendo la enfermedad marcadora de SIDA más frecuentemente diagnosticada. Desde el advenimiento del tratamiento anti-retroviral de alta eficacia (TARGA), dicha incidencia, como la de otras infecciones oportunistas, ha disminuido ${ }^{6-8}$. En la Argentina, el hallazgo de $P$. jiroveci como primera enfermedad marcadora de SIDA está en descenso, llegando en el año 2000 a menos de $15 \%$, luego de haber alcanzado su pico máximo de $32,3 \%$ en 1993. No se conoce la incidencia de infecciones por $P$. jiroveci en pacientes inmunosuprimidos con serología negativa para VIH en este país ${ }^{9,10}$.

El inicio de los síntomas, el patrón radiológico, la gravedad y mortalidad varían de acuerdo a la fisiopatología de la inmunosupresión ${ }^{3,11-15}$. En el caso de la infección por VIH, la alteración principal reside en la inmunidad celular, mediada por linfocitos T LT CD4 $+{ }^{16}$. Sin embargo, en estos pacientes, existe una extensa disfunción de linfocitos B (LB) que disminuye la calidad de la respuesta humoral. La infección por VIH ocasiona hipogamaglobulinemia, activación policlonal, pérdida de memoria de células $\mathrm{B}$ y marcadores aberrantes de superficie B, impidiendo una adecuada respuesta humoral a agentes infecciosos y vacunas ${ }^{17}$. En el caso de la inmunosupresión mediada por glucocorticoides, a nivel celular, éstos inhiben el acceso de los leucocitos a los sitios de inflamación, interfiriendo con su funcionamiento, el de las células endoteliales y de los fibroblastos, y suprimiendo la producción y los efectos de factores humorales involucrados en la respuesta inflamatoria. Los LB son relativamente resistentes a los efectos inmunosupresores de los glucocorticoides, siendo
Hospital Italiano de Buenos Aires, Argentina.

Servicio de Clínica Médica (AR, CL, JS, FF, GW, MS).

Servicio de Clínica Médica, Área de Investigación en Medicina Interna (JP, DG).

Servicio de Clínica Médica, Sección de Infectología (LB).

Los autores expresan que no hay conflictos de interés al redactar el manuscrito

Financiamiento: Servicio de Clínica Médica del Hospital Italiano de Buenos Aires.

Recibido: 23 de junio de 2014 Aceptado: 6 de enero de 2015

Correspondencia a: Alejandro Rey alejandro.rey@hospitalitaliano. org.ar 
principalmente afectada la producción de anticuerpos ${ }^{16,18}$. Las alteraciones anteriormente descritas, por lo tanto, podrían implicar una respuesta inmunológica e inflamatoria diferente, con una presentación clínica y una evolución distinta en ambas poblaciones ${ }^{19}$.

Se ha reportado que los pacientes seropositivos para VIH son más jóvenes y presentan una mayor duración de los síntomas ${ }^{13,20,21}$. Asimismo, estos pacientes tienden a desarrollar fiebre, taquipnea e hipoxemia con mayor frecuencia, pero sin significancia estadística ${ }^{13,21,22}$. Sin embargo, los pacientes seronegativos para VIH muestran un peor pronóstico con mayor progresión a falla respiratoria, requerimiento de asistencia respiratoria mecánica (ARM) y mortalidad, no alcanzando esta última una diferencia estadísticamente significativa ${ }^{19,20,22-26}$.

A pesar de las observaciones realizadas en estudios previos, la infección respiratoria por $P$. jiroveci en pacientes seronegativos para VIH continúa siendo una patología poco caracterizada y sub-diagnosticada. Debido al bajo número de pacientes incluidos en análisis previos ha sido difícil alcanzar diferencias estadísticamente significativas y cierta información ha resultado contradictoria. Por otra parte, los requerimientos terapéuticos y la evolución a falla multiorgánica no han sido evaluados hasta el momento.

El propósito de la realización del presente estudio es comparar las características clínicas, radiológicas y los hallazgos de laboratorio de la infección respiratoria por $P$. jiroveci en pacientes con infección por VIH con respecto a seronegativos para $\mathrm{VIH}$, evaluar los requerimientos terapéuticos, la evolución clínica, la respuesta al tratamiento instaurado y determinar la gravedad y mortalidad de dicha infección en ambas poblaciones.

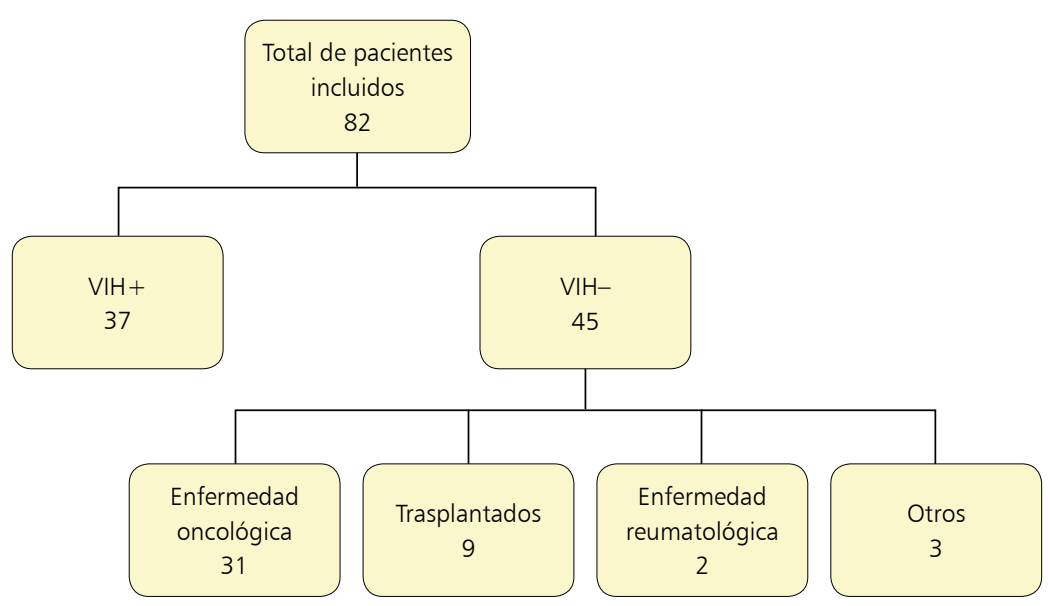

Figura 1. Pacientes incluidos en el análisis.

\section{Materiales y Métodos}

Se realizó un estudio de cohorte retrospectivo de pacientes con infección respiratoria por $P$. jiroveci con diagnóstico de infección por VIH confirmada o con inmunosupresión de otra causa. La población bajo estudio estuvo constituida por pacientes mayores de 18 años con diagnóstico de infección por $P$. jiroveci, incluyendo a todos aquellos con signos y síntomas compatibles con infección respiratoria y rescate del microorganismo en esputo y/o lavado bronquio-alveolar (LBA) por técnicas de tinción de Weigert o Giemsa y búsqueda de ADN de $P$. jiroveci por RPC (reacción de polimerasa en cadena) durante el período 2006 a 2013 en el Hospital Italiano de la ciudad de Buenos Aires. Se realizó el seguimiento de los mismos hasta el alta hospitalaria.

El muestreo fue consecutivo. Se realizó una revisión de historias clínicas de los pacientes en la que se mantuvo el anonimato de los mismos y la confidencialidad de los datos. Los datos se volcaron en una planilla de recolección confeccionada para tal fin. Se evaluaron las co-morbilidades a través del Score de Charlson ${ }^{14}$, la profilaxis antimicrobiana previa, las características clínicas, imagenológicas y de laboratorio, la evolución de la enfermedad y los requerimientos terapéuticos. Las fallas orgánicas se definieron según la $\mathrm{ACCP}^{15}$.

Análisis estadístico. Se presentan las variables continuas expresadas en mediana e intervalo intercuartil (ICC) según distribución observada y las variables categóricas como proporciones. Se compararon las medianas con el test de Mann-Whitney y las proporciones entre ambos grupos con el test de $\chi^{2}$ o exacto de Fisher según supuestos. En el análisis estadístico se utilizó el software SPSS versión 17.0. Se tomó como estadísticamente significativa las probabilidades menores a 0,05 .

\section{Resultados}

Se incluyeron 82 pacientes con infección respiratoria por $P$. jiroveci. Treinta y siete (45\%) fueron seropositivos para VIH y los 45 (55\%) restantes presentaban inmunosupresión de otras causas (Figura 1).

Con respecto al grupo seropositivo para VIH, 15 pacientes $(40,5 \%)$ presentaban diagnóstico previo de infección por $\mathrm{VIH}$, pero sólo 10 de ellos se encontraban en tratamiento con TARV. En los 22 pacientes restantes $(59,5 \%)$, la neumonía por $P$. jiroveci fue la infección oportunista que motivó el diagnóstico de infección por VIH/SIDA. La mediana de CD4 fue de 37 céls. $/ \mathrm{mm}^{3}$ (intervalo intercuartil 14-68).

Del total de pacientes seronegativos para VIH, 24 $(53,3 \%)$ recibían tratamiento con corticosteroides, y 25 
$(55,5 \%)$ recibían otra medicación inmunosupresora. Las características basales de los pacientes se presentan en la Tabla 1.

En ambos grupos, los síntomas más frecuentes fueron fiebre, disnea y en menor proporción tos irritativa, con una saturación al ingreso $\leq 92 \%$ en $43,2 \%$ de los pacientes con infección por VIH y $56,3 \%$ de los seronegativos para VIH (Tabla 2).

Los hallazgos vinculados al compromiso radiológico se describen en la Tabla 3 y los del laboratorio en la Tabla 4. Se confirmó el diagnóstico citológico en 79 pacientes. En los tres restantes se documentó la presencia de $P$. jirovec $i$ por RPC observándose en la citología cambios compatibles con respuesta inflamatoria aguda, sin documentarse otro agente etiológico.

En LBA o esputo, se documentó co-infección respiratoria con Staphylococcus aureus en cuatro pacientes, con citomegalovirus (CMV) en tres, y uno con Klebsiella pneumoniae, uno Aspergillus sp y otro con Cryptococcus neoformans.

En cuanto a la profilaxis, $13 \%$ de los pacientes con infección por VIH recibía cotrimoxazol, mientras que lo hacía $8,9 \%$ del grupo seronegativo para VIH.

Todos los pacientes fueron tratados con cotrimoxazol, debiendo rotar el mismo a pentamidina en un solo caso por alteración del hepatograma e hiponatremia. El tratamiento con cotrimoxazol se inició en 94,6\% de los integrantes del grupo con infección por VIH desde el ingreso y $88 \%$ recibió glucocorticoides, mientras que $80 \%$ de los pacientes seronegativos para $\mathrm{VIH}$ fue medicado con cotrimoxazol en forma precoz y $98 \%$ recibió glucocorticoides.

Con respecto a la utilización de inotrópicos, requerimiento de ARM y evolución a falla multiorgánica, se encontraron diferencias estadísticamente significativas entre ambos grupos, siendo mayor en el grupo seronegativo para VIH (Tabla 5). La mortalidad resultó mayor en este grupo, alcanzando significancia estadística, y siendo intrahospitalaria en todos los casos.

En el grupo con infección por VIH fallecieron tres pacientes (dos muertes atribuibles a la infección por $P$. jiroveci y una a otras causas). En el grupo seronegativo para VIH, fallecieron 15 pacientes (nueve muertes atribuibles a $P$. jiroveci y seis a otros motivos). Cabe aclarar que las causas de mortalidad distintas de la infección por $P$. jiroveci fueron complicaciones de la internación en UCI, siendo ésta motivada inicialmente por la infección respiratoria.

\section{Discusión}

Es extensa la literatura médica que describe las manifestaciones clínicas y la evolución de la infección
Tabla 1. Características basales en pacientes con neumonía por $P$. jiroveci. Comparación entre pacientes con y sin infección por VIH. Hospital Italiano, Bs. Aires. Años 2006-2013

\begin{tabular}{|c|c|c|c|}
\hline & $\begin{array}{l}\text { VIH positivo } \\
(\mathrm{n}=37)\end{array}$ & $\begin{array}{l}\text { VIH negativo } \\
\quad(n=45)\end{array}$ & Valor $p$ \\
\hline Edad* $^{*}$ & $37,46( \pm 9,21)$ & $52,47( \pm 17,56)$ & 0,001 \\
\hline Género femenino & $70 \%(26)$ & $29,5 \%(11)$ & 0,243 \\
\hline Puntaje de Charlson** & $6(4-7)$ & $2(2-4)$ & $<0,001$ \\
\hline \multicolumn{4}{|l|}{ Co-morbilidades } \\
\hline Tabaquismo & $32,4 \%(12)$ & $28,9 \%(13)$ & 0,073 \\
\hline EPOC & $2,2 \% \quad(1)$ & $5,4 \% \quad(2)$ & 0,586 \\
\hline Enfermedad reumatológica & $0 \%(0)$ & $6,7 \% \quad(2)$ & 0,248 \\
\hline Lupus eritematoso sistémico & - & $2,2 \% \quad(1)$ & \\
\hline Síndrome antifosfolipídico & - & $2,2 \% \quad(1)$ & \\
\hline Enfermedad renal & $5,4 \%(2)$ & $31,3 \%(14)$ & 0,003 \\
\hline Trasplante & $0 \% \quad(0)$ & $20 \% \quad(9)$ & 0,004 \\
\hline Renal & - & $15,5 \% \quad(7)$ & \\
\hline Hepático & - & $2,2 \% \quad(1)$ & \\
\hline Cardíaco & - & $2,2 \% \quad(1)$ & \\
\hline Enfermedad oncológica & $2,7 \%(1)$ & $68,2 \%(31)$ & $<0,001$ \\
\hline Tumores sólidos & $0 \% \quad(0)$ & $15,4 \% \quad(7)$ & \\
\hline Onco-hematológica & $2,7 \%(1)$ & $52,8 \%(24)$ & \\
\hline Linfoma no Hodgkin & $2,7 \%(1)$ & $37,4 \%(17)$ & \\
\hline Linfoma de Hodgkin & - & $2,2 \% \quad(1)$ & \\
\hline Mieloma múltiple & - & $4,4 \% \quad(2)$ & \\
\hline LLC & - & $2,2 \% \quad(1)$ & \\
\hline LLA & - & $2,2 \% \quad(1)$ & \\
\hline Síndrome mieloproliferativo crónico & - & $2,2 \% \quad(1)$ & \\
\hline Síndrome mielodisplásico & - & $2,2 \% \quad(1)$ & \\
\hline Diálisis & $5,4 \%(2)$ & $8,9 \% \quad(4)$ & 0,658 \\
\hline
\end{tabular}

Todas las variables en porcentaje (cantidad de observaciones); *Media ( \pm desvío estándar); ** Mediana (intervalo intercuartil). EPOC: Enfermedad pulmonar obstructiva crónica. LLC: Leucemia linfática crónica. LLA: Leucemia linfática aguda.

Tabla 2. Signos y síntomas en pacientes con neumonía por $P$. jiroveci. Comparación entre pacientes con y sin infección por VIH. Hospital Italiano, Bs. Aires. Años 2006-2013

\begin{tabular}{|lccc|} 
& $\begin{array}{c}\text { VIH positivo } \\
\text { (n: } \mathbf{3 7})\end{array}$ & $\begin{array}{c}\text { VIH negativo } \\
\text { (n: } \mathbf{4 5})\end{array}$ & Valor $\mathbf{p}$ \\
Disnea de reposo & $75,7 \%(28)$ & $60 \% \quad(27)$ & 0,133 \\
Tos irritativa & $75 \%(27)$ & $57,5 \%(13)$ & 0,108 \\
Fiebre & $91,9 \%(34)$ & $83,3 \%(35)$ & 0,322 \\
Deterioro del sensorio & $0 \quad(0)$ & $2,2 \%(1)$ & 1 \\
Semiología respiratoria & & & \\
$\quad$ Crépitos & $36,1 \%(13)$ & $40 \% \quad(16)$ & 0,727 \\
$\quad$ Sibilancias & $10,8 \%(4)$ & $7,1 \%(3)$ & 0,700 \\
$\quad$ Semiología bilateral & $38,9 \%(14)$ & $36,6 \%(15)$ & 1 \\
$\quad$ Uso de músculos accesorios & $22,9 \%(8)$ & $15,8 \%(6)$ & 0,444 \\
\hline Variables en porcentaje (cantidad de observaciones). & & \\
\hline
\end{tabular}


Tabla 3. Compromiso radiológico en neumonía por $P$. jiroveci. Comparación entre pacientes con y sin infección por VIH. Hospital Italiano, Bs. Aires. Años 2006-2013

\begin{tabular}{|c|c|c|c|}
\hline & $\begin{array}{l}\text { VIH positivo } \\
\text { (n: } 37)\end{array}$ & $\begin{array}{l}\text { VIH negativo } \\
\text { (n: 45) }\end{array}$ & Valor $p$ \\
\hline Radiografía de tórax & (n: 36) & (n: 41) & \\
\hline Compromiso radiológico & $88,9 \%(32)$ & $80,5 \%(33)$ & 0,311 \\
\hline Infiltrado bilateral difuso & $75,9 \%(27)$ & $70,7 \%(29)$ & 0,675 \\
\hline Presencia de efusión pleural & $5,6 \% \quad(2)$ & $2,4 \% \quad(1)$ & 0,596 \\
\hline \multicolumn{4}{|l|}{ Consolidación } \\
\hline Unilateral & $13,9 \% \quad(5)$ & $9,8 \% \quad(4)$ & 0,726 \\
\hline Bilateral & $0 \% \quad(0)$ & $0 \% \quad(0)$ & - \\
\hline Tomografía computada & $(n=32)$ & $(n=42)$ & \\
\hline Opacidades en vidrio esmerilado & $87,5 \%(28)$ & $81 \%(34)$ & 0,449 \\
\hline Opacidades difusas & $28,1 \% \quad(9)$ & $28,6 \%(12)$ & 0,966 \\
\hline
\end{tabular}

Tabla 4. Resultados de laboratorio en neumonía por $P$. jiroveci. Comparación entre pacientes con y sin infección por VIH. Hospital Italiano, Bs. Aires. Años 2006-2013

\begin{tabular}{|lccc|} 
& $\begin{array}{c}\text { VIH positivo } \\
\text { (n: } \mathbf{3 7})\end{array}$ & $\begin{array}{c}\text { VIH negativo } \\
\text { (n: } \mathbf{4 5})\end{array}$ & Valor $\mathbf{p}$ \\
\hline Valor de LDH (UI/ml) & $307(253-482)$ & $349(242-476)$ & 0,757 \\
\hline Leucocitos totales (céls./mm³) & $7.430(5.500-8.930)$ & $7.950(3.710-11.558)$ & 0,766 \\
\hline Saturación al ingreso $\leq 92 \% *$ & $43,2 \%(16)$ & $56,3 \%(18)$ & 0,281 \\
\hline $\begin{array}{l}\text { Variables expresadas en mediana (intervalo intercuartil). *Porcentaje (cantidad de observaciones). } \\
\text { LDH: Lactato deshidrogenasa. Valor de referencia LDH: 208-378 Ul/ml. }\end{array}$
\end{tabular}

Tabla 5. Gravedad y evolución en nemonía por $P$. jiroveci. Comparación entre pacientes con y sin infección por VIH. Hospital Italiano, Bs. Aires. Años 2006-2013

\begin{tabular}{|lccr}
\hline & $\begin{array}{c}\text { VIH positivo } \\
\text { (n: } \mathbf{3 7 )}\end{array}$ & $\begin{array}{c}\text { VIH negativo } \\
\text { (n:45) }\end{array}$ & Valor $\mathbf{p}$ \\
\hline Estadía hospitalaria* & $8(5-10)$ & $16 \quad(7-24)$ & 0,001 \\
\hline Cuidados intensivos & $32,4 \%(12)$ & $57,9 \%(26)$ & 0,022 \\
$\quad$ Días UCl* & $4(3-7)$ & $10(5-20)$ & 0,257 \\
Necesidad de ARM & $10,8 \%(4)$ & $46,7 \%(21)$ & $<0,001$ \\
Utilización de inotrópicos & $8,3 \%(3)$ & $40,9 \%(18)$ & 0,001 \\
Requerimiento de oxígeno & $66,7 \%(24)$ & $79,5 \%(35)$ & 0,193 \\
Falla orgánica múltiple & $8,3 \%(3)$ & $42,2 \%(19)$ & $<0,001$ \\
Disfunción SNC & $0 \%(0)$ & $4,4(2)$ & 0,194 \\
Disfunción hematológica & $5,6 \%(2)$ & $26,7(12)$ & 0,013 \\
Disfunción renal & $8,1 \%(3)$ & $42,2 \%(19)$ & 0,001 \\
Disfunción pulmonar & $8,1 \%(3)$ & $44,4 \%(20)$ & $<0,001$ \\
Disfunción hepática & $10,8 \%(4)$ & $13,3 \%(6)$ & 0,728 \\
Disfunción cardiovascular & $8,1 \%(3)$ & $37,8(17)$ & 0,002 \\
Disfunción gastrointestinal & $0 \%(0)$ & $2,2 \%(1)$ & 1 \\
Mortalidad & $8,3 \%(3)$ & $33,3 \%(15)$ & 0,007 \\
\hline Todas & & $(0)$ &
\end{tabular}

Todas las variables en porcentaje (cantidad de observaciones); *Mediana (intervalo intercuartil). UCI: unidad de cuidados intensivos. ARM: asistencia respiratoria mecánica. SNC: sistema nervioso central. respiratoria por $P$. jiroveci en pacientes infectados por VIH. Se ha reportado un aumento en la incidencia de dicha entidad en pacientes con otras causas de inmunosupresión, presentando diferencias en el comportamiento clínico y pronóstico $^{1,3}$.

Con respecto a las características basales de las dos poblaciones de pacientes que evaluamos, la diferencia estadísticamente significativa en el puntaje de comorbilidades de Charlson se debió a que dicho índice asigna un puntaje de seis a los pacientes con síndrome de inmunodeficiencia asociada a VIH (VIH/SIDA). El objetivo por el cual se decidió incluir esta medición en nuestro trabajo fue evaluar la existencia concomitante de otros factores co-mórbidos distintos del SIDA.

En el grupo con infección por VIH existió un predominio de mujeres (70\%), en contraste con la epidemiología del resto de Sudamérica y de otros centros en nuestro país, donde el diagnóstico de infección respiratoria por $P$.jiroveci en pacientes con infección por VIH/SIDA es más frecuente en el género masculino. Esta diferencia puede explicarse porque nuestro centro es un hospital privado que atiende población con cobertura médica y controles periódicos de salud. La mayoría de los pacientes portadores de VIH en seguimiento en nuestro Centro son hombres que tienen sexo con hombres. Este grupo constituye una sub-población con elevado nivel de conciencia de enfermedad, lo que contribuye al diagnóstico temprano de la infección por VIH y el tratamiento antirretroviral oportuno, como así los controles posteriores y la profilaxis primaria para $P$. jiroveci. Esto no ocurre con las mujeres, en quienes se suele arribar al diagnóstico en estadios avanzados, con bajo recuento de LTCD4 y por lo tanto, mayor susceptibilidad a presentar enfermedades oportunistas como la infección por $P$. jiroveci.

Los trabajos publicados con anterioridad al nuestro, encontraron una mayor incidencia de fiebre y disnea en pacientes seronegativos para VIH. En nuestro estudio se observó una tendencia similar, aunque sin diferencias estadísticamente significativas. A su vez, no logramos corroborar un pródromo de mayor duración en la población infectada con VIH antes descrito, debido a la naturaleza retrospectiva de nuestro trabajo ${ }^{3,4,15,21,22,24-26}$.

En cuanto al laboratorio, se han constatado niveles más altos de lactato deshidrogenada sérica (LDH) y mayor grado de desaturación en el grupo de los pacientes seronegativos para VIH, traduciendo un mayor grado de injuria pulmonar. Estos resultados concuerdan con los descritos por Su y cols., sin alcanzar relevancia estadística alguna en los estudios ${ }^{22,26}$.

El patrón radiológico típico de la infección por $P$. jiroveci consiste en un infiltrado intersticial perihiliar bilateral, descrito como "en vidrio esmerilado", en ocasiones acompañado de lesiones quísticas. Tasaka y cols., describieron que dichas imágenes son predominantes 
en los pacientes con infección por VIH presentándose en $60 \%$ de los casos. Los hallazgos radiológicos en los pacientes seronegativos para VIH son variables, pudiendo presentarse como infiltrados o consolidaciones con compromiso uni o bilateral, lo cual conlleva una mayor dificultad para el diagnóstico y la posibilidad de retraso del mismo ${ }^{22,24}$. En el presente estudio, se observó que las imágenes radiológicas típicas son más frecuentes en pacientes infectados con VIH, aunque las diferencias entre ambos grupos fueron no significativas.

Con respecto a la gravedad de la patología, en los pacientes seronegativos para VIH se observó mayor requerimiento de soporte ventilatorio mecánico e inotrópicos, siendo esta diferencia estadísticamente significativa. Dichas diferencias podrían estar vinculadas al mayor grado de respuesta inflamatoria en el grupo sin infección por VIH, asociada a injuria pulmonar y falla respiratoria ${ }^{1922,24,25}$. La mayor mortalidad en el grupo de seronegativos para VIH coincide con los hallazgos publicados ${ }^{15,19-21,26}$, logrando nuestro trabajo diferencias estadísticamente significativas, no presentes en dichos estudios.

En 1984, Kovac y cols., compararon el hallazgo de CMV en piezas de autopsia de pacientes con infección por VIH y en individuos con otras causas de inmunosupresión, revelando que la co-infección era menos común en este último grupo. Krajicek y cols., demostraron que la infección conjunta con CMV no implica mayor gravedad de la sintomatología ni reducción en la sobrevida. Resta definir el posible rol de la infección con CMV como factor predisponente para desarrollar neumonía por $P$. jiroveci en individuos inmunosuprimidos ${ }^{3,11,13}$.

La principal limitación de nuestro estudio radica en el carácter retrospectivo del mismo, que implica una mayor cantidad de datos perdidos e imprecisos, lo cual podría representar un sesgo de información. Los pacientes fueron reclutados de una población con cobertura de salud, en su mayoría incluidos en un sistema de médicos de cabecera, con seguimiento y foco en prevención. Esta característica podría no ser representativa de la población portadora de VIH en nuestro país, lo cual puede limitar la validez externa de los hallazgos.

A diferencia de estudios previos, en nuestro análisis hemos demostrado diferencias estadísticamente significativas en lo que concierne al requerimiento de ARM, evolución a falla multiorgánica y mortalidad. Comparamos asimismo variables no evaluadas en trabajos previos, como la estadía promedio en UCI, el requerimiento de inotrópicos y el desarrollo de disfunciones orgánicas.

Cabe destacar, a su vez, que la escasez de publicaciones de similar naturaleza tanto en Argentina como en Latinoamérica en general, nos permite sentar ciertas bases para estudios de mayor alcance.

La infección respiratoria por $P$. jiroveci presenta diferencias en el comportamiento clínico y en la evolución en pacientes con infección por VIH con respecto a individuos con otras causas de inmunosupresión. Debido al aumento de la población en riesgo para adquirir esta patología en pacientes seronegativos para VIH, es de fundamental importancia conocer las características de la presentación en dicha población con el objetivo de optimizar la prevención y el tratamiento de esta entidad.

\section{Resumen}

Introducción: La infección respiratoria por Pneumocystis jiroveci constituye una patología oportunista frecuente en pacientes infectados con virus de inmunodeficiencia humana (VIH), con recuentos de LTCD4 $<200$ céls. $/ \mathrm{mm}^{3}$. Sin embargo, también se han reportado casos en pacientes con otras causas de inmunosupresión. Objetivos: Comparar las características clínicas, gravedad y mortalidad de la infección respiratoria por $P$. jiroveci en pacientes con y sin infección por VIH. Materiales y Métodos: Cohorte retrospectiva, con seguimiento intrahospitalario, de pacientes adultos con infección respiratoria por $P$. jiroveci entre 2006 y 2013. Resultados: Se incluyeron 82 pacientes con infección respiratoria por $P$. jiroveci, de los cuales $55 \%$ (45) fueron seronegativos para VIH. De éstos, 68,8\%\% (31) padecía enfermedad oncológica y $20 \%$ (9) eran receptores de trasplantes. El $57,9 \%$ (26) se internó en una unidad de cuidados intensivos, 42,2\% (19) presentó falla multiorgánica (FMO), 46,7\% (21) requirió Asistencia Respiratoria Mecánica (ARM) y 40,9\% (18) inotrópicos. La mortalidad fue del $33,3 \%$ (15). Se observaron diferencias estadísticamente significativas entre ambos grupos en la edad $(p<0,001)$, requerimiento de $\mathrm{ARM}(\mathrm{p}<0,001)$ e inotrópicos ( $\mathrm{p} 0,001)$ y FMO $(\mathrm{p}<0,001)$. La mortalidad resultó mayor en el grupo seronegativo para VIH, alcanzando significancia estadística (p 0,007). Conclusión: La mortalidad fue mayor en pacientes no infectados con VIH, presentando mayor número de complicaciones y evolución a insuficiencia respiratoria con FMO. 


\section{Referencias bibliográficas}

1.- Azoulay É, Lemiale V, Kouatchet A, Roux A, Bollée G, Roux P. Pulmonary involvement in patients with hematological malignancies. Azoulay E, ed. 2011: 313-25.

2.- $\quad$ Li J, Huang X M, Fang W G, Zeng X J. Pneumocystis carinii pneumonia in patients with connective tissue disease. J Clin Rheumatol 2006; 12 (3): 114-7.

3.- Sepkowitz K A. Pneumocystis carinii pneumonia among patients with neoplastic disease. Semin Respir Infect 1992; 7 (2): 114-21.

4.- Thomas Jr. C F, Limper A H. Pneumocystis pneumonia. N Engl J Med 2004; 350 (24): 2487-98.

5.- Roblot F, Godet C, Le Moal G, Garo B, Fao M. Analysis of underlying diseases and prognosis factors associated with Pneumocystis carinii pneumonia in immunocompromised HIVnegative patients. Eur J Clin Microbiol Infect Dis 2002; 21 (7): 523-31.

6.- Palacios R, Hidalgo A, Reina C, de la Torre M, Márquez M, Santos J. Effect of antiretroviral therapy on admissions of HIV-infected patients to an intensive care unit. HIV Med 2006; 7 (3): 193-6.

7.- Pérez E, Toibaro J J, Losso M H. HIV patient hospitalization during the pre and post-HAART era. Medicina (B. Aires) 2005; 65 (6): 482-8.

8.- Tellez I, Barragán M, Franco-Paredes C, Petraro P, Nelson K, Del Río C. Pneumocystis jiroveci pneumonia in patients with AIDS in the inner city: a persistent and deadly opportunistic infection. Am J Med Sci 2008; 335 (3): 192-7.

9.- Krajicek B J, Limper A H, Thomas Jr C F. Advances in the biology, pathogenesis and identification of Pneumocystis pneumonia. Curr Opin Pulm Med 2008; 14 (3): 228-34.

10.- Boletín sobre SIDA en la República Argentina. 2000; 19.

11.- De Lorenzo L J, Huang C T, Maguire G P,
Stone D J. Roentgenographic patterns of Pneumocystis carinii pneumonia in 104 patients with AIDS. Chest 1987; 91 (3): 323-7.

12.- Gruden J F, Huang L, Turner J, Webb W R, Merrifield C, Stansell J D, et al. High-resolution $\mathrm{CT}$ in the evaluation of clinically suspected Pneumocystis carinii pneumonia in AIDS patients with normal, equivocal, or nonspecific radiographic findings. Am J Roentgenol 1997; 169 (4): 967-75.

13.- Kovacs J A, Hiemenz J W, Macher A M, Stover D, Murray H W, Shelhamer J, et al. Pneumocystis carinii pneumonia: a comparison between patients with the acquired immunodeficiency syndrome and patients with other immunodeficiencies. Ann Intern Med 1984; 100 (5): 663-71.

14.- Limper A H, Offord K P, Smith T F, Martin 2nd W J. Pneumocystis carinii pneumonia. Differences in lung parasite number and inflammation in patients with and without AIDS. Am Rev Respir Dis 1989; 140 (5): 1204-9.

15.- Sepkowitz K A. Opportunistic infections in patients with and patients without acquired immunodeficiency syndrome. Clin Infect Dis 2002; 34 (8): 1098-107.

16.- Boumpas D T, Chrousos G P, Wilder R L, Cupps T R, Balow J E. Glucocorticoid therapy for immune-mediated diseases: basic and clinical correlates. Ann Intern Med 1993; 119 (12): 1198-208.

17.- Shen X, Tomaras G D. Alterations of the B-cell response by HIV-1 replication. Curr. HIV/AIDS Rep 2011; 8 (1): 23-30.

18.- Cupps T R, Gerrard T L, Falkoff R J, Whalen G, Fauci A S. Effects of in vitro corticosteroids on $\mathrm{B}$ cell activation, proliferation, and differentiation. J Clin Invest 1985; 75 (2): 754-61.

19.- Enomoto T, Azuma A, Kohno A, Kaneko K, Saito H, Kametaka M, et al. Differences in the clinical characteristics of Pneumocystis jirovecii pneumonia in immunocompromized patients with and without HIV infection. Respirology 2010; 15 (1): 126-31.

20.- McKinnell J A, Cannella A P, Kunz D F, Miller L G, Baddley J W, Pappas P G. Pneumocystis pneumonia in hospitalized patients: a detailed examination of symptoms, management, and outcomes in human immunodeficiency virus (HIV)-infected and HIV-uninfected persons. Transpl Infect Dis 2012; 14 (5): 510-8

21.- Nüesch R, Bellini C, Zimmerli W. Pneumocystis carinii pneumonia in human immunodeficiency virus (HIV)-positive and HIV-negative immunocompromised patients. Clin Infect Dis 1999; 29 (6): 1519-23.

22.- Ewig S, Bauer T, Schneider C, Pickenhain A, Pizzulli L, Loos U, et al. Clinical characteristics and outcome of Pneumocystis carinii pneumonia in HIV-infected and otherwise immunosuppressed patients. Eur Respir J 1995; (8): $1548-53$

23.- Mansharamani N G, Garland R, Delaney D, Koziel H. Management and outcome patterns for adult Pneumocystis carinii pneumonia, 1985 to 1995: comparison of HIV-associated cases to other immunocompromised states. Chest 2000; 118 (3): 704-11.

24.- Tasaka S, Tokuda H, Sakai F, Fujii T, Tateda K, Johkoh T, et al. Comparison of clinical and radiological features of Pneumocystis pneumonia between malignancy cases and acquired immunodeficiency syndrome cases: a multicenter study. Intern Med 49 (4): 273-81.

25.- Tasaka S, Tokuda H. Pneumocystis jirovecii pneumonia in non-HIV-infected patients in the era of novel immunosuppressive therapies. J Infect Chemother 2012: 793-806.

26.- Su Y S, Lu J J, Perng C L, Chang F Y. Pneumocystis jirovecii pneumonia in patients with and without human immunodeficiency virus infection. J Microbiol Immunol Infect 2008; 41 (6): 478-82. 